\title{
Cell cycle regulation of mitochondrial protein import revealed by genome-scale pooled
}

\section{bimolecular fluorescence complementation screening}

Kim Blakely*§, Patricia Mero*, Roland Arnold*, Ayesha Saleem ${ }^{\dagger}$, Christine Misquitta*, Dahlia

Kasimer*, Sachin Kumar*§, Andrea Uetrecht*, Kevin A. Brown*, Alessandro Datti ${ }^{\ddagger}$, David Hood $^{\dagger}$, Philip Kim*§ and Jason Moffat*§1

*Donnelly Centre and Banting \& Best Department of Medical Research, §Department of Molecular Genetics, University of Toronto, 160 College Street, Toronto, Canada, M5S3E1;

${ }^{\dagger}$ Muscle Health Research Centre, York University, Farquharson Life Sciences Building Room 302, 4700 Keele Street, Toronto, Canada, M3J1P3; ${ }^{\ddagger}$ Samuel Lunenfeld Research Institute, 600 University Avenue, Toronto, Canada, M5G1X5

${ }^{1}$ Corresponding Author: University of Toronto, 160 College Street Room 802, Toronto, Canada, M5S3E1. Email: j.moffat@utoronto.ca 


\section{Running Title}

Pooled BiFC reveals TOMM22 interactome

\section{Keywords}

Bimolecular fluorescence complementation, Lentivirus, TOMM22, Protein kinase CK2, Protein-

protein interaction

\section{Corresponding Author}

Dr. Jason Moffat

University of Toronto

160 College Street Room 802

Toronto, ON, Canada

M5S3E1

Email: j.moffat@utoronto.ca

Telephone: (416) 978-0336 


\section{ABSTRACT}

A central focus of systems biology is the functional mapping of protein-protein interactions under physiological conditions. Here we describe MaGiCaL-BiFC, a lentivirus-based bimolecular fluorescence protein-fragment complementation approach for the high-throughput, genomescale identification of protein-protein interactions in mammalian cells. After developing and validating this methodology using known protein-protein interaction pairs, we constructed genome-scale pooled BiFC libraries using the human ORFeome cDNA collection. These pooled libraries, containing 12,000 unique human cDNAs, were used to screen for candidate interaction partners of the mitochondrial transmembrane protein TOMM22. Following infection of cells with the TOMM22 bait and the pooled cDNA libraries, cells harboring candidate TOMM22 interacting proteins were isolated from the cell pool via fluorescence activated cell sorting, and identified via microarray analysis. This approach identified several known interaction partners of TOMM22, as well as novel physical and functional partners that link the mitochondrial network to proteins involved in diverse cellular processes. Notably, protein kinase CK2 was identified as a novel physical interaction partner of human TOMM22. We found that this association occurs preferentially during mitosis and involves direct phosphorylation of TOMM22, an event that may lead to attenuation of mitochondrial protein import. Together, this data contributes to the growing body of evidence suggesting eloquent coordination between cell cycle progression and mitochondrial physiology. Importantly, through high-throughput screening and focused validation, our study demonstrates the power of the MaGiCaL-BiFC approach to uncover novel functional protein-protein interactions, 
bioRxiv preprint doi: https://doi org/10.1101/770669; this version posted September 16,2019 . The copyright holder for this preprint (which was not certified by peer review) is the author/funder, who has granted bioRxiv a license to display the preprint in perpetuity. It is made available under aCC-BY-NC-ND 4.0 International license.

including those involving proteins with membrane-spanning domains, or of a transient nature, all within their native cellular environment. 


\section{INTRODUCTION}

Currently, new protein-protein interactions (PPIs) are most commonly identified using yeast two-hybrid (Y2H) methods (Fields and Song 1989; Rual et al. 2005) or affinity purification followed by mass spectrometry (AP-MS) (Ewing et al. 2007; Mak et al. 2010). While such techniques have offered great insight into the PPI networks in some mammalian cell types, there are disadvantages inherent to these methodologies. Notably, both technologies face severe limitations when trying to examine the interaction profiles of membrane-bound proteins. In recent years, development of the protein fragment complementation assay (PCA), including the biomolecular fluorescence complementation (BiFC) approach, have reformed the study of protein-protein interactions in vivo (Hu et al. 2002; Remy and Michnick 2004; Kerppola $2008 \mathrm{~b})$. These assays are based on the observation that the two halves of a rationally dissected protein can reconstitute in vivo to create a functional protein. Such technologies have been applied with success in mammalian cells using transfection-based methodologies (Hu et al. 2002; Remy and Michnick 2004), as well as an enhanced retroviral mutagen technique (Ding et al. 2006). Despite this, application of these technologies remains limited as transfection-based techniques are constrained to use in transfection-amenable cell types, and are often subject to high false positive rates, while the retroviral method faces limitations in terms of applicability to a wide range of mammalian cell types and the lack of full-length transcripts generated using this technology. Although no technique is likely to provide a perfect solution to these issues, an in vivo mammalian-based system bridging recent advances in both pooled-barcoded library screening and lentiviral-based technologies would help provide a solution to a number of these issues. Lentiviral expression systems encoding in-frame fusions to full-length human genes can 
be easily introduced into virtually any mammalian cell type, and expression levels off lentiviral vectors are more easily controlled compared to transfection-based techniques.

To this end, we have designed MaGiCaL-BiFC: a Mammalian, Gateway-Compatible (Walhout et al. 2000), Lentiviral-based (Zufferey et al. 1997; Dull et al. 1998; Moffat et al. 2006), Bimolecular Fluorescence protein-fragment Complementation assay (Michnick et al. 2000; Remy et al. 2002; Michnick 2003; Remy et al. 2007a; Remy et al. 2007b; Remy and Michnick 2007; Kerppola 2009; Schutze et al. 2009) based on the reconstitution of a split fluorescent protein, Venus (Nagai et al. 2002), for the genome-scale analysis of PPIs in mammalian cells. In MaGiCaL-BiFC, the two fragments of Venus are tagged to proteins of interest using Gateway recombinant technology (Hartley et al. 2000), packaged into lentivirus, and stably expressed as fusions within cells. When expressed separately, the split Venus fragments do not form a functional fluorophore (Kerppola 2008a). However, when each fragment is fused to one protein of an interacting pair, Venus is reconstituted creating a stable and quasi-irreversible fluorescent complex (Lalonde et al. 2008). The advantages of MaGiCaL-BiFC over currently used methodologies to study PPIs in mammalian cells are numerous: PPIs are detected in vivo, occur in their native cellular compartment, and most importantly, their localization can be visualized via microscopy, providing powerful insight into the biological function of specific PPIs. The lentiviral nature of this system allows for pooled genome-wide screening of PPIs in any cell line of interest using fluorescence activated cell sorting (FACS) (Mak et al. 2011). In addition, the pooled genetic screening approach involves minimal cost and is compatible with sequencing or microarray de-convolution. 


\section{MATERIALS AND METHODS}

\section{Cell lines}

HEK293T cells were cultured in Dulbecco's modified Eagle's medium (DMEM) supplemented with $10 \%$ heat-inactivated fetal bovine serum (iFBS) and $1 \%$ penicillin/streptomycin (pen-strep) (Wisent, St. Bruno, QC). HeLa cells were cultured in McCoy's 5A medium supplemented with $10 \%$ fetal bovine serum (FBS) and 1\% pen-strep. The ReNcell VM NSC line was obtained from Millipore (Billerica, MA) and cultured according to the manufacturer's protocol. Fibroblast growth factor (FGF) and epidermal growth factor (EGF) were purchased from BD Biosciences (Bedford, MA).

\section{Antibodies}

Primary antibodies against TOMM22 (ab57523), TOMM20 (ab56783), CSNK2A1 (ab70774), MYC-HRP (ab1326) and FLAG-HRP (ab49763) were from Abcam (Cambridge, MA). HRPconjugated secondary antibodies for Western blot detection were from Cell Signaling Technologies (NEB; Ipswich, MA). Primary antibodies against cyclins (sampler kit 9869) and for co-IP detection of CSNK2A1 (2656) were from Cell Signaling Technologies.

\section{Construction of the MaGiCaL-BiFC destinations vectors}

The MaGiCaL-BiFC destination vectors were generated by PCR amplification of the CMV promoter and Gateway cassette with flanking N- and C-terminal Venus fragments from the vectors RFB-VF1, RFB-VF2, VF1-RFA and VF2-RFA (generous gift of Dr. Stephen Michnick) and cloned into pLM1 (Sancak et al. 2008) using SnaBI and BstBI restriction sites. The bait vectors 
were further modified by the addition of an IRES-RFP-WPRE fragment cloned between the $\mathrm{Xcm}$ I and Nsil sites to replace the PGK promoter and PAC resistance gene.

\section{Proof-of-concept localization studies}

Full-length cDNAs were obtained from the human ORFeome v5.1 entry clone collection (Thermo Fisher, Open Biosystems) and sequence verified. Gateway LR reactions were performed according to the manufacturer's protocol (Life Technologies, Carlsbad, CA) to introduce the cDNA inserts into the MaGiCaL-BiFC destination vectors. Lentivirus was used to infect HEK293T and ReNcell VM NSC cultures at an MOI of 1. Four-days post-infection the cells were imaged via confocal microscopy. For NSC differentiation, bFGF and EGF were removed from the growth media four-days post-infection and cells were allowed to differentiate for one week. Media was changed every two days.

\section{Construction of the MaGiCaL-BiFC hORFeome v5.1 libraries}

The hORFeome v5.1 entry clone library was pooled into 43 sub-pools of 370 clones each, and each pool was standardized to a concentration of $150 \mathrm{ng} / \mu \mathrm{L}$. A standard $\mathrm{LR}$ reaction using the prey vectors, pLD-CMVpr-VF2-Gateway-hPGKpr-puro and pLD-CMVpr-Gateway-VF2-hPGKprpuro, was performed with each pool for a total of $86 \mathrm{LR}$ reactions. Following proteinase $\mathrm{K}$ inactivation, each reaction was transformed into ElectroMAX DH5-alpha_E cells (Life Technologies) and plated on LB agar $+100 \mu \mathrm{g} / \mathrm{mL}$ ampicillin. Overnight incubation at $37^{\circ} \mathrm{C}$ generated $\sim$ 400,000 colonies per transformation, for a 1000-fold representation of each pool. Colonies from each individual plate were pooled, and plasmid DNA extracted using a QIAfilter 
Plasmid Maxi Prep kit (Qiagen, Valencia, CA). DNA was standardized to $500 \mathrm{ng} / \mu \mathrm{L}$, and pooled together to generate separate $\mathrm{N}$ - and C-terminal MaGiCaL-BiFC libraries.

\section{Lentivirus production}

For the VF1-TOMM22 and prey library virus, ten million cells were seeded into $150 \mathrm{~mm}$ dishes twenty-four hours prior to transfection in DMEM supplemented with $10 \%$ iFBS and $0.1 \%$ penstrep. The bait or prey-library plasmid DNA (18 $\mu \mathrm{g})$, along with the packing plasmid psPAX2 $(16.2 \mu \mathrm{g})$ and envelope plasmid pMD2.G $(1.8 \mu \mathrm{g})$ were transfected using Fugene 6 (Roche, Basel, Switzerland) according to the manufacturer's protocol. Eighteen hours post-transfection media was replaced with DMEM containing $1.1 \%$ bovine serum albumin (BSA) and 1\% penstrep. Virus was harvested at $42 \mathrm{~h}$ and $66 \mathrm{~h}$ post-transfection, pooled, aliquoted and stored at $80^{\circ} \mathrm{C}$. Small-scale virus preparations were prepared in $60 \mathrm{~mm}$ plates using area-adjusted reagents.

\section{The MaGiCaL-BiFC pooled-screening procedure}

HEK293T cells were co-transduced with VF1-TOMM22 and either the NVF2- or CVF2-prey library virus at a MOI of 0.5 each, such that $25 \%$ of cells contained both a bait and prey construct. Twenty-four hours post-infection the cells were selected with $2 \mu \mathrm{g} / \mathrm{mL}$ puromycin (Wisent). Four-days post-infection the cells were washed once with warm PBS, and collected by trypsinization. Cells were resuspended in PBS/2\% iFBS containing 2 mM EDTA and counted. One million cells were pelleted and frozen at $-80^{\circ} \mathrm{C}$ as a pre-sort background sample, and the remaining cells were analyzed on a BD Facsaria, sorting for $\mathrm{RFP}^{+}$Venus ${ }^{+}$events. For the $\mathrm{N}-$ 
terminal library screens, approximately 5 million cells were sorted of which $27.36 \%+/-3.78 \%$ were RFP+ and $0.079 \%+/-0.042 \%$ were RFP+/Venus+, providing a 125-fold coverage of the library ORFs screened. For the C-terminal library screens, approximately 3 million cells were sorted of which $25.89 \%+/-1.26 \%$ were RFP+ and $0.069 \%+/-0.012 \%$ were RFP+/Venus+, providing a 75-fold coverage of the library ORFs screened (Table S1 and Figure S4). Sorted cells were imaged post-sort on a WaveFX confocal spinning disc microscope (Quorum Technologies) using standard filter sets for FITC (Venus) and TexasRed (RFP) to confirm Venus expression in the sorted cells. Sorted cells were expanded for one week in culture before harvesting.

\section{Identification of positive hits from sorted populations}

Genomic DNA was isolated from the expanded cells, and unsorted control populations, using the DNeasy Blood and Tissue Kit (Qiagen). The cDNA inserts were PCR-amplified using primers specific for either the $\mathrm{N}$ - or C-terminal libraries using Phusion DNA polymerase (New England Biolabs, Ipswich, MA). Amplicons were PCR-purified using the QIAquick PCR Purification Kit

(Qiagen), and subsequently labeled using the BioPrime DNA Labeling System (Invitrogen).

Biotinylated probes were hybridized to GeneChip Human Gene 1.0 ST Arrays (Affymetrix, Santa Clara, CA) according to the manufacturer's protocol. Chip files were analyzed using Affymetrix Expression Console Software using hORFeome5-1 analysis file and RMA-Sketch normalization for analysis, with results in log form. The 3 replicates of the $\mathrm{N}$-terminal dataset were analyzed together, and the background chip was analyzed separately. Similar analysis was done for Cterminal sets. The resulting files were then exported as .txt files using the annotation merge file 
hORFeome.hugene-1_0-st.v1_na28_hg18. The microarray includes 15,347 probesets that measure expression levels of 12,556 Entrez Gene IDs. For each of the screens, array expression values were floored at 1 , and probesets were filtered to remove those with high average background from duplication measurements (bg > 1000) or low median expression from triplicate experiments (expr < 200). For the remaining probesets, the ratio of the expression intensity to background intensity was computed, along with the median and median absolute deviation (MAD) of the ratios. Hits were defined as probesets with ratios that were more than three MADs above the median value. For N-terminal screens, this threshold was 2.24, while for the C-terminal screens the threshold was 1.73. There was 294 probesets representing 263 unique Entrez Gene IDs called hits in the N-terminal screen, while there was 152 probesets representing 130 unique Entrez Gene IDs in the C-terminal screen (Table S2).

\section{Pair-wise BiFC validation}

Full-length cDNA clones for select putative interaction partners of TOMM22 were picked from the human ORFeome v5.1 collection and sequence validated. Clones were transferred into the MaGiCaL-BiFC destination vectors by LR site-specific recombination, and used to generate lentivirus. HEK293T cells were co-transduced with VF1-TOMM22 and the select baits at a MOI of 1-5. Four days post-infection, cells were imaged on a WaveFX spinning disc confocal microscope using standard filter sets for FITC (Venus) and TexasRed (RFP).

\section{Secondary siRNA screen}


The siRNA secondary screen was performed by transfecting HEK293T cells seeded in 384-well plates with Dharmacon siRNA SMARTpools, with each pool containing 4 siRNAs against a given target. Each transfection was performed in duplicate. Three days post-transfection, cells were stained with MitoTracker Green FM, MitoTracker Red CMXRos, and Hoechst 33342. Plates were imaged using a GE Healthcare IN Cell Analyzer 2000 epifluorescent HTS microscope. Images were acquired using a 10X long working-distance $0.45 \mathrm{NA}$ objective (Nikon) and standard filter sets for FITC (MitoTracker Green FM), Cy3 (MitoTracker Red CMXRos) and DAPI (Hoechst). Nine fields of view per well were captured. Images were analyzed using Perkin Elmer's Columbus image analysis server running Acapella 2.0. Reported values represent the average from duplicate wells.

\section{Protein extraction and immunoblotting}

Cells were lysed with RIPA lysis buffer containing protease inhibitor cocktail (Sigma) and quantified by BCA (Pierce). Lysates (10-40 $\mu \mathrm{g} /$ lane) were separated by SDS-PAGE and protein was transferred onto a polyvinylidene fluoride (PVDF) membrane (Hybond-P, GE Healthcare, Uppsala, Sweden). Specific primary antibodies (see Antibodies subsection) were detected using the appropriate secondary HRP-conjugated antibodies and visualized by enhanced chemiluminescence detection (Supersignal West Pico; Pierce - Thermo Fisher Scientific).

\section{Exogenous co-immunoprecipitation experiments}

Full-length cDNA clones for TOMM34 and CSNK2A1 were picked from the human ORFeome v5.1 collection and sequence verified. Clones were transferred into a N-terminal MYC-tagged 
lentiviral destination vector by LR site-specific recombination. N-terminal FLAG-tagged

TOMM22 was generated by LR site-specific recombination of the TOMM22 entry clone into the

MAPLE lentiviral destination vector (Mak et al. 2010). HEK293T cells were co-transfected with

TOMM22 and the select baits, and were subsequently immunoprecipitated using $\alpha$ FLAG M2-

conjugated agarose beads (Sigma-Aldrich, St. Louis, MO). Bound proteins were resolved by

SDS-PAGE and detected using an aMYC-tag HRP-conjugated antibody (Abcam).

\section{Cell cycle blocks}

HeLa cells were seeded in $150 \mathrm{~mm}$ plates in normal growth media at a density of 2.5 million cells/plate. Twenty-four hours post-seeding, media was exchanged for media containing $4 \mathrm{mM}$ thymidine (Sigma-Aldrich). Following an overnight incubation (16 h), cells were released into fresh media for $7.5 \mathrm{~h}$. Media was again exchanged for media containing $4 \mathrm{mM}$ thymidine or $200 \mathrm{ng} / \mathrm{mL}$ nocodazole (Sigma-Aldrich). Following an overnight incubation (16 h), cells were released into fresh media for varying amounts of time. Release of double thymidine-blocked cells for $0,2.5,5.5$ and $7.5 \mathrm{~h}$ generated synchronous cell populations at approximately G1-S, early S, late S and S-G2 phases of the cell cycle. Release of thymidine/nocodazole blocked cells for 0.5 and $3 \mathrm{~h}$ generated synchronous cell populations at approximately $\mathrm{M}$ and $\mathrm{G} 1$ phases of the cell cycle. Cells were collected to prepare lysates for Western blot analysis and confirmation of the cell cycle phases using specific primary antibodies against Cyclin A, Cyclin B1, Cyclin E2 or Cyclin E (Cell Signaling Technology), or for endogenous co-IPs. 


\section{Endogenous IP of TOMM22 and protein kinase CK2 in asynchronous and M-phase blocked} cells

Asynchronously growing HeLa cells and HeLa cells blocked in M-phase were harvested in co-IP buffer (50 mM Hepes-KOH, 0.1\% Triton-X100, 2 mM EDTA, 100 mM NaCl, and 1X protease inhibitors (Sigma-Aldrich)) and lysed for 30 minutes on ice. Lysates were cleared by centrifugation at $14,000 \mathrm{~g}$ for 10 minutes at $4^{\circ} \mathrm{C}$. Lysates were quantified by $\mathrm{BCA}$ and equal amounts of asynchronous or M-phase lysates were used for the IP of endogenous TOMM22 or CSNK2A1 using specific primary antibodies (see Antibodies subsection) overnight at $4^{\circ} \mathrm{C}$ with rotation. The next day protein G Ultralink beads (Pierce-Thermo Fisher Scientific) were added to the samples, which continued to rotate for an additional $2 \mathrm{~h}$ at $4{ }^{\circ} \mathrm{C}$. Beads were collected by centrifugation at 2,200 RPM, and washed 3 times with ice cold PBS. Bound proteins were resolved by SDS-PAGE, and used for subsequent Western blotting for detection of immunoprecipitated TOMM22 and CSNK2A1.

\section{Generation of phosphorylation site mutations in TOMM22}

Mutagenesis primers were used to introduce phosphorylation site mutations into the TOMM 22 entry clone using the GeneArt Site-Directed Mutagenesis System (Life Technologies). Primers specific to serine residue 15 and threonine residue 43 were each individually mutated to alanine (A) and glutamic acid (E). In addition, the double alanine (AA) and double glutamic acid (EE) mutants were generated. Common primers were used to amplify these ORFs to introduce a single N-terminal FLAG tag into the construct, as well as an in-frame stop codon. These entry clones were then introduced into the pMAL vector (generous gift of L. Naldini) by site-specific 
LR reaction, and virus was generated as described above. HEK293T cells were transduced with the lentiviral constructs, or a control Renilla luciferase (RLUC) construct designed with an Nterminal FLAG tag. Two-days post infection, cells were infected with an shRNA targeting the 3'UTR of TOMM22 to deplete the endogenous TOMM22 transcript.

\section{Mitochondrial isolations}

Mitochondrial isolations were performed as described previously (Frezza et al. 2007). Briefly, five $150 \mathrm{~mm}$ tissue-culture dishes were seeded with 10 million cells for each of the mutant cell lines two days prior to the experiment. On the day of isolation, cells ( 200 million per mutant) were washed twice with ice-cold PBS, and collected using a cell scraper. Cells were pelleted at $600 \mathrm{~g}$ at $4^{\circ} \mathrm{C}$ for 10 minutes, resuspended in $3 \mathrm{~mL}$ ice-cold $\mathrm{IB}_{\mathrm{c}}$ buffer ( $200 \mathrm{mM}$ sucrose, $10 \mathrm{mM}$ Tris-MOPS pH 7.4, 100 nM EGTA/Tris) and homogenized using a Teflon pestle/glass potter operated at 1500 RPM for 30 strokes. Nuclei were pelleted by centrifugation at $600 \mathrm{~g}$ at $4{ }^{\circ} \mathrm{C}$ for 10 minutes. The supernatant was collected and pelleted at $7000 \mathrm{~g}$ at $4^{\circ} \mathrm{C}$ for 10 minutes. The mitochondrial pellet was washed once with ice-cold IB $\mathrm{B}_{c}$ buffer, and a fraction was taken for lysis in RIPA buffer, followed by quantification by BCA.

\section{In vitro transcription, translation and import of OCT}

The in vitro mitochondrial import assays were performed as described previously (Singh and Hood 2011). Briefly, vector encoding full-length ornithine transcarbamylase (OCT) was linearized using Sacl and subsequently phenol extracted and ethanol precipitated. OCT was in vitro transcribed at $40^{\circ} \mathrm{C}$ for 90 min using SP6 RNA polymerase, followed by in vitro translation 
in the presence of $\left[{ }^{35} \mathrm{~S}\right]$ methionine within a rabbit reticulocyte lysate system. For the mitochondrial protein import assay, $50 \mu \mathrm{g}$ of mitochondria were preincubated for $10 \mathrm{~min}$ at $30^{\circ} \mathrm{C}$ before the import assay. Translation mix $(12 \mu \mathrm{l})$ was added to the mitochondria, and the import incubation was allowed to proceed at $30^{\circ} \mathrm{C}$ for $8 \mathrm{~min}$. Subsequently, import was halted by the addition of an aliquot of the mitochondrial translation mix to an ice-cold sucrose cushion (600 mM sucrose, $100 \mathrm{mM} \mathrm{KCl,} 20 \mathrm{mM} \mathrm{HEPES}$, and $2 \mathrm{mM} \mathrm{MgCl}$ ). Mitochondria were pelleted by centrifugation for $15 \mathrm{~min}$ at $16,000 \mathrm{~g}$ at $4^{\circ} \mathrm{C}$ and resuspended in $20 \mu \mathrm{l}$ of ice-cold breaking buffer (600 mM sorbitol, $20 \mathrm{mM}$ HEPES, $\mathrm{pH}$ 7.4). The samples were then denatured at $95^{\circ} \mathrm{C}$ in the presence of lysis buffer for 5 min, quick cooled on ice, and then resolved by SDS-PAGE (12\% gel). Gels were processed and dried as described elsewhere (Craig et al. 1998). Images and subsequent quantification were obtained with electronic autoradiography (Quantity One, BioRad, Hercules, CA). Imported mature OCT (MOCT) was distinguished from precursor OCT (pOCT) by its lower molecular weight. The percentage of imported protein was calculated based on the ratio of the intensity of the mOCT to total OCT protein (mOCT+pOCT). A Repeated Measures ANOVA was used to evaluate the significance of the difference between the $A$ and $E$ mutants. Each mutant was evaluated in three biologically independent experiments, following fresh lentiviral infections and mitochondrial isolations.

\section{RESULTS}

\section{Design of the MaGiCaL-BiFC system}

We designed MaGiCaL-BiFC to enable genome-wide analyses of PPIs in any mammalian cell line of interest using any bait protein of interest, based on the bimolecular fluorescence protein- 
fragment complementation assay and lentiviral-based pooled barcode screening methodology.

To achieve this, a series of lentiviral (Zufferey et al. 1997; Dull et al. 1998) destination vectors were developed that incorporate a Gateway expression cassette (Walhout et al. 2000) in frame with either the $\mathrm{N}$ - or C-terminal fragment of the rationally-dissected fluorescent protein Venus (VF1 and VF2, respectively) (Nagai et al. 2002; MacDonald et al. 2006). To circumvent issues that may arise due to orientation of the tag, vectors were built in both orientations, such that cDNAs could be tagged on either terminus. The bait vectors (containing VF1) include an internal ribosome entry site (Van Der Kelen et al. 2009) in frame with red fluorescent protein (IRES-RFP) to gauge bait expression levels. Prey vectors (containing VF2) include the puromycin resistance gene puromycin $\mathrm{N}$-acetyltransferase (PAC) under control of the constitutive human phosphoglycerate kinase promoter (hPGKpr-puro) to allow efficient selection of preyexpressing cells (Figure 1A).

To test the sensitivity and accuracy of MaGiCaL-BiFC, we generated expression clones for established protein interactions with specific localization patterns (ie. "test set") including FXDY1-ATP1B1 (plasma membrane), NUPL1-NUP62 (nuclear membrane), TOMM22-TOMM20 (mitochondria), CYP1A1-CYB5A (endoplasmic reticulum), RPL9-RPS3 (ribosome/cytoplasmic) and CDK4-CCND3 (nuclear). Vectors were generated by Gateway site-specific recombination, with one protein of the pair introduced into both orientations of the bait vector, and the other into both orientations of the prey vector. Lentiviruses from these expression constructs were generated and used to infect HEK293T cells either singly, as pairs (testing all four possible combinations) or with a negative control construct that includes beta-galactosidase (LACZ). Venus signal was undetectable by fluorescence microscopy when each bait-VF1 or prey-VF2 
construct was expressed alone, and was extremely faint and/or uneven with the complementary negative control construct. When the correct pairs were expressed together, predicted signal was readily detectable by either microscopy (Figure 1B and Figure S1) or flow cytometry (Figure 1C).

To further assess our lentiviral system in cell types that are difficult to transfect, we infected human neural stem cells (ie. ReNcell VM cells), with the lentivirus test assays described above, and found the predicted Venus localization patterns (Figure S2A). Moreover, these stem cells were differentiated into neural cells by removing mitogens (bFGF and EGF) for one week, and then re-imaged (Figure S2B). Signal intensity and specificity were maintained in the differentiated neural cells, addressing the applicability of this system to cell types refractory to transfection-based PPI detection methods such as neural stem cells.

\section{Construction of the MaGiCaL-BiFC libraries}

We next wanted to develop prey libraries to enable pooled genome-scale PPI screens by virtue of the fact that reconstituted Venus signal can be efficiently detected and sorted by FACS for many different protein-protein interaction assays (Figure 1C). To generate the $\mathrm{N}$ - and Cterminal VF2 lentiviral pooled libraries, the human ORFeome v5.1 entry clone library was pooled and introduced into the prey destination vectors by Gateway site-specific recombination to generate individual lentiviral-based expression plasmid pools with VF2 sequence in frame with either the $\mathrm{N}$ - or $\mathrm{C}$-terminus of $>12,000$ unique human open reading frames (ORFs) (Figure S3A). Since each human ORF can act as a barcode, a long-range PCR procedure was developed with common amplification primers to generate labeled probe from 
the expression plasmid pools, as well as genomic DNA from HEK293T cells infected with the pooled lentivirus libraries in order to assess representation and quality of the ORFs postinfection. We estimated library representation to be $>70 \%$ in the lentiviral plasmid pools and following packaging and infection into HEK293T cells (Figure S3B).

\section{Proof-of-concept screen for TOMM22 binding partners}

We chose to investigate the PPI network of TOMM22, which plays a central role in cell metabolism, survival, and growth (Yano et al. 2000; Bellot et al. 2007). TOMM22 was an attractive target since it forms a complex with several additional components of the TOM complex, mediating protein translocation into mitochondria. Due to the orientation of the TOMM proteins in the outer mitochondrial membrane (OMM) (Figure 2A), only specific tagging orientations should generate positive fluorescent signal when these interactions are assessed by MaGiCaL-BiFC, as was observed in the pairwise tests described above (Figure 1B), providing an ideal scheme to test the methodology.

To screen for TOMM22 interacting proteins with pools of VF2-tagged preys using cell sorting and microarray de-convolution, the VF1-TOMM22 construct, which has the VF1-tag facing the cytoplasm, was generated for use as the bait (Figure 2A). To ensure that the protein expression off the lentiviral constructs was not exaggerated relative to endogenous levels, which could lead to high false positive rates, the protein levels of VF1-TOMM22, and its known interaction partner TOMM20-VF2 (described above), were analyzed by Western blot following transduction into HEK293T cells and found to be similar or lower than endogenous TOMM22 and TOMM20 (Figure 2B), consistent with what has been observed for similar lentiviral vector 
systems (Mak et al. 2010). For screening, HEK293T cells were simultaneously infected with the VF1-TOMM22 bait virus and either the $\mathrm{N}$ - or C-terminally tagged prey virus libraries at a multiplicity of infection of $\sim 0.5$ for each of the bait and prey, so that an average of $25 \%$ of the cells expressed both the bait and a prey construct. For the $\mathrm{N}$-terminal library screens, approximately 5 million cells were sorted of which $27.36 \%+/-3.78 \%$ were RFP+ and $0.079 \%+/-$ 0.042\% were RFP+/Venus+, providing a 125-fold coverage of the library ORFs screened (Table S1 and Figure S4). For the C-terminal library screens, approximately 3 million cells were sorted of which $25.89 \%+/-1.26 \%$ were RFP+ and $0.069 \%+/-0.012 \%$ were RFP+/Venus+, providing a 75-fold coverage of the library ORFs screened (Table S1 and Figure S4). Genomic DNA was harvested from the sorted cells, as well as a control population of unsorted cells, in order to amplify the ORF inserts using common primers that flank the Gateway cassette. Following amplicon labeling, samples were hybridized to oligonucleotide microarrays and subsequently scanned to identify enriched signals that represent putative TOMM22 interactors (Figure 2C). In order to identify putative TOMM22 interacting partners, we developed an enrichment score $r$, the median ratio of probeset intensities to background for each gene, where a hit was defined as $r$ greater than 3 MADs above the median. This equated to 263 and 130 unique genes from the $\mathrm{N}$-terminal and C-terminal library screens, respectively (Table S2). The analysis revealed a number of expected positive controls; for example TOMM20, a wellcharacterized TOMM22 interactor, was identified as the top hit in the C-terminal screen $(r=45.5)$, consistent with its orientation in the OMM (Figure 2D). Conversely, TOMM5 ( $r=6.7)$ and TOMM6 ( $r=9.2)$ were only detected in the $\mathrm{N}$-terminal screen, as expected (Figure $2 \mathrm{E})$. In addition to identifying several TOM complex proteins such as TOMM20, TOMM5 and TOMM6, 
we also identified additional OMM proteins related to apoptosis (e.g. BAX, BCL2L1, BCL2L2 and BCL2L13), mitochondrial morphology (e.g. FIS1), cytoskeletal function (e.g. CORO1B, PFN1, TWF20) and metabolism (e.g. MGLL, FBP2, G6PD, CDS2, LDHB, CYB5B) (Table S2). Notably, the pro-apoptotic BCL2-family member BAX is a well-characterized interaction partner of TOMM22 (Bellot et al. 2007), and was specifically identified in the N-terminal MaGiCaL-BiFC screen, consistent with previous reports on its mitochondrial membrane orientation (Suzuki et al. 2000).

To confirm a subset of putative VF1-TOMM22 orientation-specific interactions from the primary screening data, we constructed a selection of prey constructs tagged with VF2 at the Nand C-termini and tested these in bimolecular fluorescence complementation assays in HEK293T cells using the MaGiCaL-BiFC vectors. Proteins for follow-up were selected based on interest and prior knowledge, and were chosen to include those with variable functions and subcellular localization patterns. For instance, mitochondrial import and mitochondrial morphology have been shown to be tightly coordinated processes, although the mechanisms regulating this are only poorly understood (Stojanovski et al. 2006). Given that FIS1, which is known to regulate mitochondrial morphology, was identified as a strong TOMM22 interactor, we chose to pursue this interaction in follow-up. Other mitochondrial proteins, including FUNDC1 and FUNDC2 were chosen based on the principle that members of this family are newly implicated in autophagy (Liu et al. 2012). Given that the TOMM machinery has been shown to function in this process (Bertolin et al. 2013; Yang and Yang 2013), we thought that these interactions might help to shed additional light on this important cellular process. Similarly, crosstalk between mitochondria and endoplasmic reticulum is an emerging area of 
study that leaves much to be uncovered (English and Voeltz 2013; Kornmann 2013). For that reason, we chose to investigate the putative endoplasmic reticulum proteins identified as interaction partners of TOMM22, including both HMOX1 and HMOX2. Other putative interaction partners such as GALE, LIN28, and TMEM79 were chosen based on the fact that they have little to no known prior information linking them to TOMM22, or mitochondria, and novel interactions with TOMM22 may help to provide additional insight into their biological functions. In total, 17 genes were tagged in both orientations and 14/17 showed the predicted localization pattern (Figure 3A). As expected, the TOMM5 and TOMM6 preys showed a strong fluorescence signal in the mitochondria with the bait TOMM22 in an orientation-specific manner (Figure $3 \mathrm{~A})$. The remaining 12 preys that showed a strong Venus fluorescence signal included FIS1, FAM82A2, HMOX1, HOMX2, FBP1, FBP2, GALE, LIN28, FUNDC1, FUNDC2, TMEM79 and REEP6 (Figure 3A). The Venus signals also co-localized with the mitochondrial stain Mitotracker Red CMXRos (Figure 3B). Notably, the co-localization patterns varied in that some interactions showed strong OMM staining (e.g. HMOX1, HMOX2) while others showed punctate mitochondrial staining (e.g. FAM82A2/RMD3). Overall, these observations suggest that our primary TOMM22 sorting screen revealed a number of known and potentially novel protein interactions with a component of the TOM complex.

\section{Link between TOMM22 interactors and mitochondrial mass/potential}

We reasoned that some of the putative novel TOMM22 interactors that were identified in our screens could also be important for mitochondrial physiology. To test this hypothesis, we performed a secondary siRNA screen to determine the effects of knocking down the TOMM22 
interaction partners on mitochondrial mass and/or potential. We chose 80 random putative interactors from both the $\mathrm{N}$ - and $\mathrm{C}$-terminal screens and used high-content microscopy and quantitative image analyses to measure the changes in mitochondrial mass and/or potential following knockdown of these genes with siRNA pools and staining with Mitotracker dyes (Figure 4A). HEK293T cells were plated in 384-well plates and transfected in duplicate with siRNA pools targeting each of the 80 putative interaction partners. siRNAs targeting SIN3A, BAK, and MFN2, which have been previously reported to influence mitochondrial membrane potential, were included as controls. A scrambled siRNA pool that has no effect on membrane potential relative to untransfected cells was also included as a control. Three days posttransfection, the cells were stained with two different mitochondria-specific dyes, MitoTracker Green FM and MitoTracker Red CMXRos, and analyzed by high-content microscopy. Importantly, while both dyes accumulate in mitochondria based on mitochondrial mass, MitoTracker Red CMXRos is also sensitive to membrane potential. Thus, the red-to-green ratio can be used to evaluate changes in mitochondrial membrane potential specifically, as has been previously reported (Yoon et al. 2010).

Target genes were classified as hits if they altered the MitoTracker Red CMXRos-toMitoTracker Green FM signal (reflecting a change in mitochondrial membrane potential) more than three standard deviations away from the mean of the siScramble control wells. The control gene, SIN3A, whose knockdown was previously reported to increase mitochondrial membrane potential (Yoon et al. 2010), came out among our top candidates that increased the MitoTracker Red CMXRos-to-MitoTracker Green FM signal ratio, indicating an increase in mitochondrial membrane potential (Figure 4B, pink bar). Also, the pro-apoptotic protein BAK 
came out among the top genes whose knockdown increased the MitoTracker Red CMXRos-toMitoTracker Green FM ratio (Figure 4B, red bar), consistent with the pro-apoptotic role of BAK in mitochondrial outer membrane permeabilization and membrane potential dissipation (Breckenridge and Xue 2004). Conversely, knockdown of MFN2 decreased mitochondrial membrane potential (Figure 4B, green bar), which is consistent with previous reports (Chen et al. 2003). Using this approach, we found that siRNA-mediated knockdown of $~ 57 \%$ of the 80 putative TOMM22 interaction partners identified in MaGiCaL-BiFC had an effect on mitochondrial membrane potential. This is in contrast to genome-scale approaches employing a similar method, which have reported that $\sim 3 \%$ of screened candidates affect membrane potential or mass when depleted (Yoon et al. 2010). This suggests that our MaGiCaL-BiFC hits are highly enriched for genes that impact mitochondrial physiology.

One of the candidates identified as a TOMM22 interaction partner, and whose knockdown in the secondary siRNA screen decreased mitochondrial membrane potential, was the protein kinase CK2 alpha (also known as CK2 $\alpha$ or CSNK2A1), encoded by the CSNK2A1 gene (Figure 4B, blue bar). Interestingly, a physical and functional interaction was recently described between yeast CK2 and yeast Tom22 (Schmidt et al. 2011), which was reported to result in Tom22 phosphorylation, leading to increased localization of Tom22 to mitochondria, and ultimately increased protein import into mitochondria. Although human TOMM22 phosphorylation has not been studied in mammalian cells, phospho-TOMM22 peptides have been reported in large-scale phospho-proteomic screens (Dephoure et al. 2008; Olsen et al. 2010). Importantly, both of these phosphorylation sites, serine 15 and threonine 43 , conform to the protein kinase CK2 phosphorylation consensus sequence, $S / T-X-X-E / D$, where protein kinase 
CK2 will phosphorylate a serine or threonine residue located $\mathrm{N}$-terminally of acidic amino acid residues (Meggio and Pinna 2003). Moreover, both occur in the cytoplasmic domain of TOMM22. Interestingly, the previous detection of phospho-TOMM22 was reported in proteome-scale phosphoproteomic studies that analyzed the phosphoproteome under varying phases of the cell cycle. Both S15 (Olsen et al. 2010) and T43 (Dephoure et al. 2008) on TOMM22 were found to be phosphorylated specifically in cells synchronized at M-phase, suggesting that these are mitotic-specific phosphorylation events.

\section{Protein kinase CK2 interacts with and can phosphorylate TOMM22}

To validate the interaction between TOMM22 and protein kinase CK2, CSNK2A1 was introduced into the N-terminally tagged MaGiCaL-BiFC prey vector, consistent with the tagging-orientation identified in the screen. VF1-TOMM22 and VF2-CSNK2A1 were co-transduced into HEK293T cells, and Venus signal was readily detectable by fluorescence microscopy compared to control infections, thus confirming the interaction between protein kinase CK2 and TOMM22 by this method (Figure 4C).

To validate the interaction between TOMM22 and protein kinase CK2 using an orthogonal protein interaction assay, co-immunoprecipitation (co-IP) experiments were performed. Briefly, TOMM22 was co-expressed in HEK293T cells as an N-terminally FLAGtagged protein together with MYC fusion proteins corresponding to negative (GFP) and positive (TOMM34) control interaction partners, as well as protein kinase CK2. FLAG-tagged TOMM22 was immunoprecipitated (IP) using $\alpha$ FLAG M2-conjugated beads, and following washing, bound proteins were separated by SDS-PAGE and Western blots were performed using an anti-MYC 
antibody for detection of the co-immunoprecipitated interactors. No interaction could be detected between TOMM22 and GFP, however TOMM22 was effectively able to co-IP both TOMM34 and protein kinase CK2 (Figure 4D).

To further investigate the endogenous interaction between TOMM22 and protein kinase CK2, a series of cell cycle blocks using both thymidine and nocodazole to arrest HeLa cells in various phases of the cell cycle were performed, followed by IP of either TOMM22 or protein kinase CK2. Efficient block and release of the cells at the various cell cycle phases was assessed through Western blot analysis of the relative levels of various cyclin proteins (Figure S5). Importantly, we observed an interaction between TOMM22 and protein kinase CK2 with reciprocal IPs at endogenous protein levels (Figure 4E), which was more prevalent when using lysates from mitosis-arrested cells (Figure 4E), consistent with previous reports on TOMM22 phosphorylation status in human cell lines (Dephoure et al. 2008; Olsen et al. 2010). Taken together, these results suggest that protein kinase CK2 physically associates with TOMM22 in the cell, and this interaction is enhanced during mitosis.

\section{TOMM22 phosphorylation by protein kinase CK2 impacts mitochondrial protein import}

We reasoned that because protein kinase CK2 associates with TOMM22, it might directly phosphorylate TOMM22. To confirm that protein kinase CK2 can directly phosphorylate TOMM22, in vitro phosphorylation assays were performed using recombinant GST-tagged TOMM22 and purified protein kinase CK2 holoenzyme in the presence of radiolabelled ATP. Protein kinase CK2 was able to effectively phosphorylate TOMM22 in a time-dependent manner (Figure 5A), suggesting that there may be a functional role for the protein kinase CK2- 
TOMM22 interaction in mammalian cells.

Given that TOMM22 is critical for protein import into mitochondria, we sought to assess if TOMM22 phosphorylation affected this process. In yeast, it has recently been shown that the phosphorylation of Tom 22 by CK2 increased Tom22 localization to the mitochondrial membrane, and stimulated TOM complex formation, resulting in increased import of TOM complex members into the OMM (Schmidt et al. 2011). The S. cerevisiae and human TOMM22 proteins have $32 \%$ sequence similarity (Yano et al. 2000), and their functions are highly conserved, suggesting together with our screen data, that the functional consequences of this interaction may also be conserved in human cells. To investigate if phosphorylation of human TOMM22 was affecting the import of proteins into human mitochondria, a series of TOMM22 mutants were generated where the reported phospho-sites in the cytoplasmic domain of TOMM22 (serine 15 and threonine 43; Figure 5B) were each individually mutated to alanine (to block phosphorylation) or glutamic acid (to mimic phosphorylation), creating the clones S15E, S15A, T43E, and T43A. Additionally, the double phospho-null (AA) and double phospho-mimic (EE) were also generated. These clones also possessed an N-terminal FLAG tag so that exogenous TOMM22 could be distinguished from the endogenous protein by Western blot analysis. Wild-type TOMM22 and a Renilla luciferase (RLUC) construct were also N-terminally FLAG-tagged and used as controls. These sequences were stably expressed using a lentiviral system in HEK293T cells. To avoid interference from endogenous TOMM22, endogenous TOMM22 was depleted in cell lines stably expressing different TOMM22 phospho-mutants with an shRNA targeting the 3'UTR of endogenous TOMM22 (Figure 5B). To determine if TOMM22 phosphorylation influences the process of mitochondrial protein import, in vitro mitochondrial 
import assays were performed to assess the import capacity of mitochondria expressing mutant versions of TOMM22. Importantly, mitochondrial isolations from the mutant cell lines, and subsequent Western blot analysis of the relative levels of TOMM22-mutant protein, revealed that the localization of mutant TOMM22 to mitochondria was not impaired (Figure 5C). This was in contrast to the yeast system, where phospho-null mutants were impaired in their ability to localize to mitochondria (Schmidt et al. 2011).

To test the import capacity of mitochondria expressing mutant TOMM22, we performed in vitro mitochondrial import assays, which assess the ability of isolated mitochondria to import an in vitro transcribed and translated precursor protein, pre-ornithine transcarbamylase (pOTC), into the mitochondrial matrix (Terada et al. 1996). If imported into mitochondria, the pre-sequence of the OCT protein is cleaved off, generating a smaller protein, which can be distinguished as a lower molecular weight species by SDS-PAGE. Thus, protein import capacity can be estimated by quantifying the amount of mature OCT relative to the total amount in the reaction. Using mitochondria isolated from each of the stable cell lines described above (Figure $5 B, C)$, it was found that the phospho-mimics were significantly impaired in the import of pOCT (Figure 5D). This is in contrast to the yeast report where phosphorylation increased import of TOM complex members and precursor proteins into mitochondria. These results indicate that the interaction between protein kinase CK2 and TOMM22 is conserved between yeast and man, but the human interaction appears to occur in a cell cycle dependent manner to decrease mitochondrial protein import into mitochondria during mitosis. 


\section{DISCUSSION}

We have described a new method, MaGiCaL-BiFC, for the sensitive and accurate detection of PPIs in mammalian cells. This system has numerous advantages over other PPI detection methodologies: (1) the lentiviral design of our system allows for the detection of PPIs in cell lines typically not amenable to high-throughput PPI screening approaches such as stem cells and terminally differentiated cell types, (2) the Gateway compatibility of our lentiviral vectors allows for the facile generation of genome-wide or cell-specific cDNA libraries using sequence-specific recombination, (3) the pooled-screening approach allows for the highthroughput analysis of PPIs in a cost-effective manner, precluding the requirement for abundant starting materials, (4) the ability to detect small proteins that may be missed by APMS techniques, (5) the irreversible folding of the fluorescent complex allows for the trapping and detection of transient interactions, or those with a weak but biologically important affinity, which other approaches would fail to detect, (6) the accurate study of native integral membrane proteins without altering localization signals, or rigorous purification schemes, and finally (7) the interaction can be localized, providing important information on the biological significance of the PPI. Importantly, MaGiCaL-BiFC provides an improvement over current mammalian BiFC methodologies, which although have been extremely valuable, have limitations with respect to the cell lines and proteins amenable to study.

We have applied MaGiCaL-BiFC to screen for TOMM22 protein interactions and have identified a list of highly enriched putative interaction partners of the TOM complex. The ease with which we were able to detect known and unknown interactors of TOMM22 highlights the applicability of this system to challenging proteins such as those with membrane-spanning 
domains. The specific identification of TOMM5 and TOMM6 in the N-terminal screens, and

TOMM20 in the C-terminal screens, highlights the accuracy of this screening approach.

Although not all of these putative interactors are likely bone fide interaction partners of

TOMM22, the ability of BiFC to detect both binary and bystander protein interactions (e.g., in

higher order complexes) (Vidi and Watts 2009) was reflected in the number of high confidence

hits identified in these screens.

The ability of MaGiCaL-BiFC to detect the interaction between TOMM22 and protein

kinase CK2 truly highlights the utility of this approach. Directed by previous phospho-proteomic data, we were able to confirm that this interaction is likely a mitotic-enriched event. This finding speaks to the effectiveness of MaGiCaL-BiFC at identifying transiently interacting proteins, which would otherwise be missed by more stringent technologies such as AP-MS.

The recent report that yeast CK2 interacts with and phosphorylates yeast Tom22 prompted us to pursue this interaction for follow-up (Schmidt et al. 2011). Interestingly, while the interaction is conserved in human cells, the consequences of this interaction are not. Importantly, in yeast, it was found that Tom 22 is constitutively phosphorylated by CK2, and removal of this modification prevented mitochondrial protein import. This resulted from decreased association of un-phosphorylated Tom22 at the outer mitochondrial membrane. In contrast, recent reports using human cell lysates synchronized at various phases of the cell cycle suggest that unlike yeast, human TOMM22 is only phosphorylated during mitosis (Dephoure et al. 2008; Olsen et al. 2010). In support of this, the interaction between endogenous human protein kinase CK2 and TOMM22 was highly enriched in cells arrested in M-phase. Additionally, although the TOMM22 phosphorylation mutants did not show any 
differences in their ability to localize to mitochondria, we found that mutants mimicking the phosphorylation of either serine 15 or threonine 43 significantly blocked mitochondrial protein import. This is in direct contrast with the findings in yeast, but in line with the Olsen et al. study, which reported through enrichment analysis of their mitotic-specific phosphorylation events, that such events on metabolic proteins would likely decrease their activity (Olsen et al. 2010).

Given the role of protein kinase CK2 in cancer progression, it is logical to hypothesize that constitutive phosphorylation of TOMM22 may contribute to the metabolic changes commonly observed in human cell cancers. Protein kinase CK2 over-expression would likely result in constitutive phosphorylation of TOMM22 in cancer cells, decreasing mitochondrial protein import, and resulting in less functional mitochondria. This would be consistent with reports that have suggested that cancer cell mitochondria are generally smaller in size (Rehman et al. 2012). Moreover, decreased mitochondrial import of proteins important for respiration could contribute to the increased rates of glycolysis typical of cancer cells, or recent discoveries linking decreased mitochondrial respiration and cellular reprogramming (Xu et al. 2013). These ideas represent interesting avenues for future follow-up.

Altogether, this study has highlighted the utility of MaGiCaL-BiFC to identify both strong and weak or transient protein-protein interactions in mammalian cells, providing strong incentive for its application to other proteins important for normal and disease biology. This technology is particularly amenable to the discovery of membrane-based protein interactions, including proteins with membrane-spanning domains, in their native cellular environment. Thorough follow-up provided physical and functional validation of a number of the hits 
identified in this proof-of-concept screen, and validation of the interaction between TOMM22 and protein kinase CK2 has validated the utility of this approach to uncover novel biology that other protein-protein interaction methodologies have failed to uncover. When combined with other methodologies including Y2H, AP-MS and current PCA methodologies, MaGiCaL-BiFC provides an additional layer of coverage that can be utilized in the effort to map the functions of human proteins.

\section{ACKNOWLEDGEMENTS}

We thank members of the Moffat laboratory for helpful discussions and I. Stagljar for comments on the manuscript. K.B. was supported by an NSERC graduate scholarship. This work was supported by funds from the Canadian Institutes for Health Research, Canadian Foundation for Innovation, Ontario Ministry of Research and Innovation and the Canadian Institute for Advanced Research to J.M. J.M. is a Tier II Canada Research Chair in Functional Genomics and Research Fellow at the Canadian Institute for Advanced Research. 


\section{LITERATURE CITED}

G. Bellot, P. F. Cartron, E. Er, L. Oliver, P. Juin et al., 2007 TOM22, a core component of the mitochondria outer membrane protein translocation pore, is a mitochondrial receptor for the proapoptotic protein Bax. Cell death and differentiation 14 (4): 785-794.

G. Bertolin, R. Ferrando-Miguel, M. Jacoupy, S. Traver, K. Grenier et al., 2013 The TOMM machinery is a molecular switch in PINK1 and PARK2/PARKIN-dependent mitochondrial clearance. Autophagy 9 (11).

D. G. Breckenridge, and D. Xue, 2004 Regulation of mitochondrial membrane permeabilization by BCL-2 family proteins and caspases. Current opinion in cell biology 16 (6): 647-652.

H. Chen, S. A. Detmer, A. J. Ewald, E. E. Griffin, S. E. Fraser et al., 2003 Mitofusins Mfn1 and Mfn2 coordinately regulate mitochondrial fusion and are essential for embryonic development. The Journal of cell biology 160 (2): 189-200.

E. E. Craig, A. Chesley, and D. A. Hood, 1998 Thyroid hormone modifies mitochondrial phenotype by increasing protein import without altering degradation. The American journal of physiology 275 (6 Pt 1): C1508-1515.

N. Dephoure, C. Zhou, J. Villen, S. A. Beausoleil, C. E. Bakalarski et al., 2008 A quantitative atlas of mitotic phosphorylation. Proceedings of the National Academy of Sciences of the United States of America 105 (31): 10762-10767. 
Z. Ding, J. Liang, Y. Lu, Q. Yu, Z. Songyang et al., 2006 A retrovirus-based protein

complementation assay screen reveals functional AKT1-binding partners. Proc Natl Acad Sci U S A 103 (41): 15014-15019.

T. Dull, R. Zufferey, M. Kelly, R. J. Mandel, M. Nguyen et al., 1998 A third-generation lentivirus vector with a conditional packaging system. Journal of virology 72 (11): 8463-8471.

A. R. English , and G. K. Voeltz, 2013 Endoplasmic reticulum structure and interconnections with other organelles. Cold Spring Harb Perspect Biol 5 (4): a013227.

R. M. Ewing, P. Chu, F. Elisma, H. Li, P. Taylor et al., 2007 Large-scale mapping of human protein-protein interactions by mass spectrometry. Mol Syst Biol 3: 89.

S. Fields, and O. Song, 1989 A novel genetic system to detect protein-protein interactions. Nature 340 (6230): 245-246.

C. Frezza, S. Cipolat, and L. Scorrano, 2007 Organelle isolation: functional mitochondria from mouse liver, muscle and cultured fibroblasts. Nat Protoc 2 (2): 287-295.

J. L. Hartley, G. F. Temple, and M. A. Brasch, 2000 DNA cloning using in vitro site-specific recombination. Genome research 10 (11): 1788-1795.

C. D. Hu, Y. Chinenov, and T. K. Kerppola, 2002 Visualization of interactions among bZIP and Rel family proteins in living cells using bimolecular fluorescence complementation. Mol Cell 9 (4): 789-798. 
T. K. Kerppola, 2008a Bimolecular fluorescence complementation (BiFC) analysis as a probe of protein interactions in living cells. Annu Rev Biophys 37: 465-487.

T. K. Kerppola, 2008b Bimolecular fluorescence complementation: visualization of molecular interactions in living cells. Methods Cell Biol 85: 431-470.

T. K. Kerppola, 2009 Visualization of molecular interactions using bimolecular fluorescence complementation analysis: characteristics of protein fragment complementation. Chem Soc Rev 38 (10): 2876-2886.

B. Kornmann, 2013 The molecular hug between the ER and the mitochondria. Curr Opin Cell Biol 25 (4): 443-448.

S. Lalonde, D. W. Ehrhardt, D. Loque, J. Chen, S. Y. Rhee et al., 2008 Molecular and cellular approaches for the detection of protein-protein interactions: latest techniques and current limitations. Plant J 53 (4): 610-635.

L. Liu, D. Feng, G. Chen, M. Chen, Q. Zheng et al., 2012 Mitochondrial outer-membrane protein FUNDC1 mediates hypoxia-induced mitophagy in mammalian cells. Nat Cell Biol 14 (2): 177-185.

M. L. MacDonald, J. Lamerdin, S. Owens, B. H. Keon, G. K. Bilter et al., 2006 Identifying offtarget effects and hidden phenotypes of drugs in human cells. Nature chemical biology 2 (6): 329-337. 
A. B. Mak, K. M. Blakely, R. A. Williams, P. A. Penttila, A. I. Shukalyuk et al., 2011 CD133 protein $\mathrm{N}$-glycosylation processing contributes to cell surface recognition of the primitive cell marker AC133 epitope. The Journal of biological chemistry 286 (47): 41046-41056.

A. B. Mak, Z. Ni, J. A. Hewel, G. I. Chen, G. Zhong et al., 2010 A lentiviral functional proteomics approach identifies chromatin remodeling complexes important for the induction of pluripotency. Mol Cell Proteomics 9 (5): 811-823.

F. Meggio, and L. A. Pinna, 2003 One-thousand-and-one substrates of protein kinase CK2? The FASEB journal : official publication of the Federation of American Societies for Experimental Biology 17 (3): 349-368.

S. W. Michnick, 2003 Protein fragment complementation strategies for biochemical network mapping. Current opinion in biotechnology 14 (6): 610-617.

S. W. Michnick, I. Remy, F. X. Campbell-Valois, A. Vallee-Belisle, and J. N. Pelletier, 2000 Detection of protein-protein interactions by protein fragment complementation strategies. Methods in enzymology 328: 208-230.

J. Moffat, D. A. Grueneberg, X. Yang, S. Y. Kim, A. M. Kloepfer et al., 2006 A lentiviral RNAi library for human and mouse genes applied to an arrayed viral high-content screen. Cell 124 (6): 1283-1298.

T. Nagai, K. Ibata, E. S. Park, M. Kubota, K. Mikoshiba et al., 2002 A variant of yellow fluorescent protein with fast and efficient maturation for cell-biological applications. Nature biotechnology 20 (1): 87-90. 
J. V. Olsen, M. Vermeulen, A. Santamaria, C. Kumar, M. L. Miller et al., 2010 Quantitative phosphoproteomics reveals widespread full phosphorylation site occupancy during mitosis. Science signaling 3 (104): ra3.

J. Rehman, H. J. Zhang, P. T. Toth, Y. Zhang, G. Marsboom et al., 2012 Inhibition of mitochondrial fission prevents cell cycle progression in lung cancer. The FASEB journal : official publication of the Federation of American Societies for Experimental Biology 26 (5): $2175-2186$.

I. Remy, F. X. Campbell-Valois, and S. W. Michnick, 2007a Detection of protein-protein interactions using a simple survival protein-fragment complementation assay based on the enzyme dihydrofolate reductase. Nat Protoc 2 (9): 2120-2125.

I. Remy, A. Galarneau, and S. W. Michnick, 2002 Detection and visualization of protein interactions with protein fragment complementation assays. Methods in molecular biology 185: 447-459.

I. Remy, G. Ghaddar, and S. W. Michnick, 2007b Using the beta-lactamase protein-fragment complementation assay to probe dynamic protein-protein interactions. Nat Protoc 2 (9): 2302-2306.

I. Remy, and S. W. Michnick, 2004 A cDNA library functional screening strategy based on fluorescent protein complementation assays to identify novel components of signaling pathways. Methods 32 (4): 381-388. 
I. Remy, and S. W. Michnick, 2007 Application of protein-fragment complementation assays in cell biology. BioTechniques 42 (2): 137, 139, 141 passim.

J. F. Rual, K. Venkatesan, T. Hao, T. Hirozane-Kishikawa, A. Dricot et al., 2005 Towards a proteome-scale map of the human protein-protein interaction network. Nature 437 (7062): 1173-1178.

Y. Sancak, T. R. Peterson, Y. D. Shaul, R. A. Lindquist, C. C. Thoreen et al., 2008 The Rag GTPases bind raptor and mediate amino acid signaling to mTORC1. Science 320 (5882): 14961501.

O. Schmidt, A. B. Harbauer, S. Rao, B. Eyrich, R. P. Zahedi et al., 2011 Regulation of mitochondrial protein import by cytosolic kinases. Cell 144 (2): 227-239.

K. Schutze, K. Harter, and C. Chaban, 2009 Bimolecular fluorescence complementation (BiFC) to study protein-protein interactions in living plant cells. Methods in molecular biology 479: 189-202.

K. Singh, and D. A. Hood, 2011 Effect of denervation-induced muscle disuse on mitochondrial protein import. Am J Physiol Cell Physiol 300 (1): C138-145.

D. Stojanovski, M. Rissler, N. Pfanner, and C. Meisinger, 2006 Mitochondrial morphology and protein import--a tight connection? Biochim Biophys Acta 1763 (5-6): 414-421.

M. Suzuki, R. J. Youle, and N. Tjandra, 2000 Structure of Bax: coregulation of dimer formation and intracellular localization. Cell 103 (4): 645-654. 
K. Terada, I. Ueda, K. Ohtsuka, T. Oda, A. Ichiyama et al., 1996 The requirement of heat shock cognate 70 protein for mitochondrial import varies among precursor proteins and depends on precursor length. Molecular and cellular biology 16 (11): 6103-6109.

K. Van Der Kelen, R. Beyaert, D. Inze, and L. De Veylder, 2009 Translational control of eukaryotic gene expression. Crit Rev Biochem Mol Biol 44 (4): 143-168.

P. A. Vidi , and V. J. Watts, 2009 Fluorescent and bioluminescent protein-fragment complementation assays in the study of $\mathrm{G}$ protein-coupled receptor oligomerization and signaling. Mol Pharmacol 75 (4): 733-739.

A. J. Walhout, G. F. Temple, M. A. Brasch, J. L. Hartley, M. A. Lorson et al., 2000 GATEWAY recombinational cloning: application to the cloning of large numbers of open reading frames or ORFeomes. Methods in enzymology 328: 575-592.

X. Xu, S. Duan, F. Yi, A. Ocampo, G. H. Liu et al., 2013 Mitochondrial regulation in pluripotent stem cells. Cell Metab 18 (3): 325-332.

J. Y. Yang, and W. Y. Yang, 2013 Bit-by-bit autophagic removal of parkin-labelled mitochondria. Nat Commun 4: 2428.

M. Yano, N. Hoogenraad, K. Terada, and M. Mori, 2000 Identification and functional analysis of human Tom22 for protein import into mitochondria. Molecular and cellular biology 20 (19): 7205-7213. 
J. C. Yoon, A. Ng, B. H. Kim, A. Bianco, R. J. Xavier et al., 2010 Wnt signaling regulates

mitochondrial physiology and insulin sensitivity. Genes \& development 24 (14): 15071518.

R. Zufferey, D. Nagy, R. J. Mandel, L. Naldini , and D. Trono, 1997 Multiply attenuated lentiviral vector achieves efficient gene delivery in vivo. Nature biotechnology 15 (9): 871-875. 


\section{FIGURE LEGENDS}

Figure 1 MaGiCaL-BiFC system. (A) Schematic of the MaGiCaL-BiFC lentiviral destination vectors containing a Gateway expression cassette in-frame with either the N- or C-terminal fragment of the fluorescent protein Venus (VF1 and VF2, respectively). The bait vectors include an internal ribosome entry site in frame with the gene encoding the red fluorescent protein (IRES-RFP). Prey vectors include a puromycin resistance gene (PAC) under the control of the constitutive human phosphoglycerate kinase promoter (hPGKpr-PURO). (B) Series of MaGiCaL-BiFC assays in different cellular compartments. Expression clones corresponding to protein-protein interaction pairs with well established cellular localization patterns were generated for the plasma membrane, FXYD domain containing ion transport regulator 1 (FXYD1) and ATPase, Na+/K+ transporting, beta 1 polypeptide (ATP1B1); ribosome, ribosomal protein L9 (RPL9) and ribosomal protein S3 (RPS3); mitochondria, translocase of outer mitochondrial membrane 22 homolog (yeast) (TOMM22) and translocase of outer mitochondrial membrane 20 homolog (yeast) (TOMM20); nuclear membrane, nucleoporin like 1 (NUPL1) and nucleoporin 62kDa (NUP62); and nucleus, cyclin-dependent kinase 4 (CDK4) and cyclin D3 (CCND3). Four-days post infection at an $\mathrm{MOI}<0.3$, cells were imaged for Venus expression. Scale bar denotes $16 \mu \mathrm{m}$. (C) HEK293T cells infected $(\mathrm{MOK}<1)$ with the indicated lentiviruses were harvested and analysed by flow cytometry for Venus expression.

Figure 2 Proof-of-concept screen with TOMM22. (A) Schematic of the mitochondrial TOM complex showing orientation of the individual components at the outer mitochondrial membrane. (B) Western blot analysis for tagged and endogenous TOMM22 and TOMM20 in 
doubly infected HEK293T cells. (C) Schematic of the MaGiCaL-BiFC screening protocol, (1)

MaGiCaL-BiFC expression clones were packaged as lentivirus particles, (2) lentivirus was collected and used to doubly infect HEK293T cells in triplicate at a MOI of 0.5 each, (3) fourdays post infection RFP+Venus ${ }^{+}$cells were collected, (4) genomic DNA was extracted from expanded cells for PCR amplification of the cDNA inserts. Following amplicon labeling, samples were hybridized to microarrays to identify putative interactors. The results of the average of the 3 microarray hybridization experiments plotted as fold-enrichment over background as a function of probeset/index for the N-terminal screens (D) and the C-terminal screens (E).

Figure 3 Confirmation of TOMM22 interactors by pairwise BiFC. (A) Confirmation of a subset of known and newly identified TOMM22 protein interactors. (B) Co-staining of TOMM22 protein interactions with Mitotracker to validate mitochondrial localization of the PCA signal.

Figure 4 TOMM22 physically interacts with protein kinase CK2. (A) Schematic of secondary screen for effects of perturbing TOMM22 interactors on mitochondrial physiology. Cells transfected in 384-well plates with siRNA pools targeting select putative TOMM22 interaction partners were stained with MitoTracker Red CMXRos (which accumulates based on mass and potential) and MitoTracker Green FM (which accumulates based on mass). The ratio of the redto-green signal is used as a relative measure of mitochondrial membrane potential. (B) Bar graph displays the genes ordered along the $\mathrm{x}$-axis from highest to lowest relative membrane potential index (calculated relative to siScramble control wells). Dashed lines indicate 3 standard deviations above and below the siScramble membrane potential index. BAK (red bar), 
SIN3A (pink bar) MFN2 (green bar) and protein kinase CK2 (blue bar) are indicated. (C)

MaGiCal-BiFC confirmation of the interaction between protein kinase CK2 and TOMM22. (D)

Over-expression co-IP confirmation of the TOMM22-CSNK2A1 interaction. HEK293T cells were

transfected with either N-terminally FLAG-tagged TOMM22 alone, or with N-terminally MYC-

tagged TOMM34, protein kinase CK2 or GFP. TOMM22 was immunoprecipitated from cell

lysates using $\alpha$ FLAG M2 agarose beads, and interactors were detected by Western blot using an

QMYC-tag antibody. (E) Endogenous co-IP of protein kinase CK2 and TOMM22 from

asynchronous and M-phase arrested cells.

Figure 5 TOMM22 phosphorylation disrupts mitochondrial protein import. (A) TOMM22 phosphorylation by protein kinase CK2 was carried out with GST-TOMM22 in the presence protein kinase CK2 for increasing amounts of time. Reactions lacking TOMM22 or protein kinase CK2 were included as controls. Proteins were resolved by SDS-PAGE and visualized by autoradiography. (B) Schematic of TOMM22 protein and location of predicted protein kinase CK2 phospho-sites. Western blot analysis of whole cell lysates showing the relative expression of the N-terminally FLAG-tagged Renilla luciferase (36 kDa) and TOMM22 WT and mutant constructs (16.5 kDa) relative to endogenous TOMM22 (15.5 kDa) protein levels. (C) Western blot analysis of TOMM22 mutant expression from mitochondrial preparations. (D) Radiography of OCT import into mitochondria. Values represent the average from three independent experiments. p, precursor OCT; m, mature OCT. Data displayed are representative of three biological replicates (independent infections and mitochondrial isolations). (E) Bar graph 
bioRxiv preprint doi: https://doi.org/10.1101/770669; this version posted September 16, 2019. The copyright holder for this preprint (which was not certified by peer review) is the author/funder, who has granted bioRxiv a license to display the preprint in perpetuity. It is made available under aCC-BY-NC-ND 4.0 International license.

showing the average relative import of OCT. Phospho-nulls and phospho-mimics have been grouped to display the effect of phosphorylation on import. 
A Bait Vectors:

pLD-CMVpr-VF1-Gateway-IRES-RFP

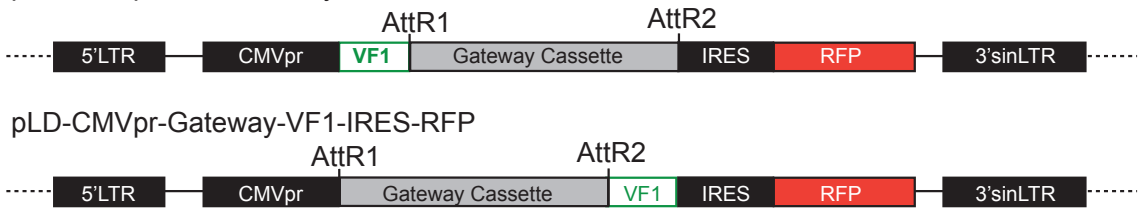

Prey Vectors:

pLD-CMVpr-VF2-Gateway-hPGKpr-Puro

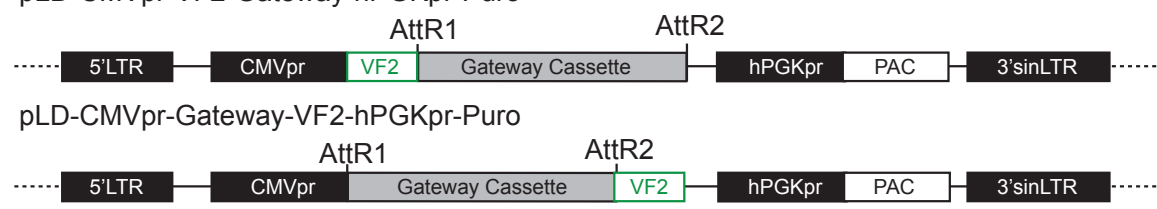

B Plasma Membrane Nuclear Membrane Mitochondria

Ribosome

Nuclear
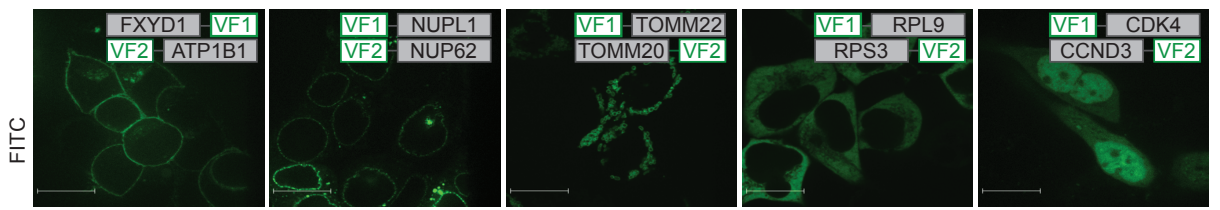

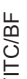
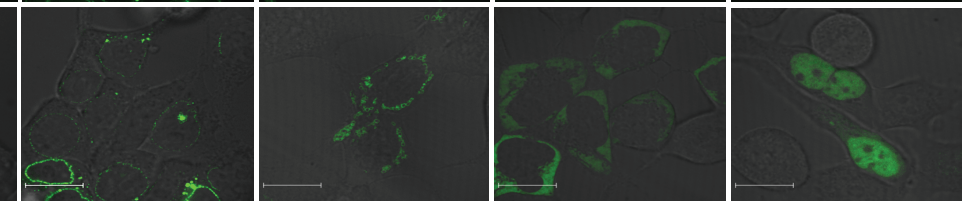

C

FXYD1-VF1

VF2-ATP1B1

FXYD1-VF1

+ VF2-LACZ

FXYD1-VF1
+ VF2-ATP1B

- VF1-NUPL1

VF2-NUP62

VF1-NUPL1

+ VF2-LACZ

FF1-TOMM22

TOMM20-VF2

VF1-TOMM22

+ LACZ-VF2

VF1-NUPL1
+ VF2-NUP62

VF1-TOMM22

+ TOMM2O-VF2

VF1-RPL9

RPS3-VF2

VF1-RPL9

+ LACZ-VF2

VF1-RPL9

+ RPS3-VF2

VF1-CDK4

CCND3-VF2

VF1-CDK4

+ LACZ-VF2

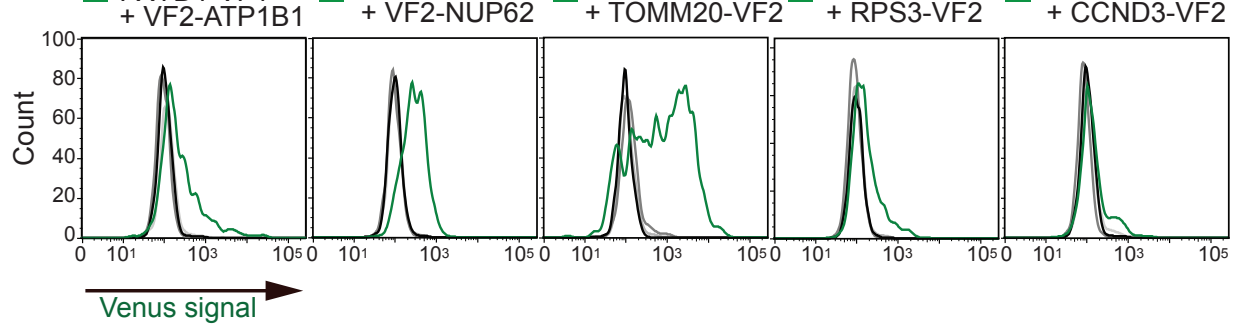


Figure 2

A

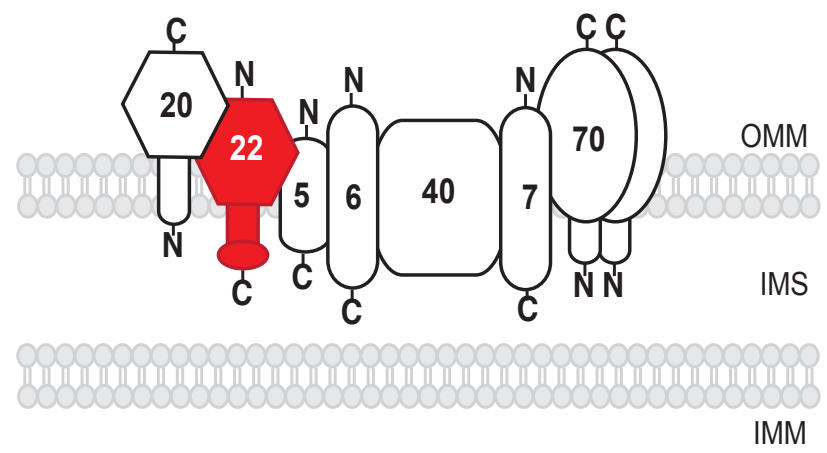

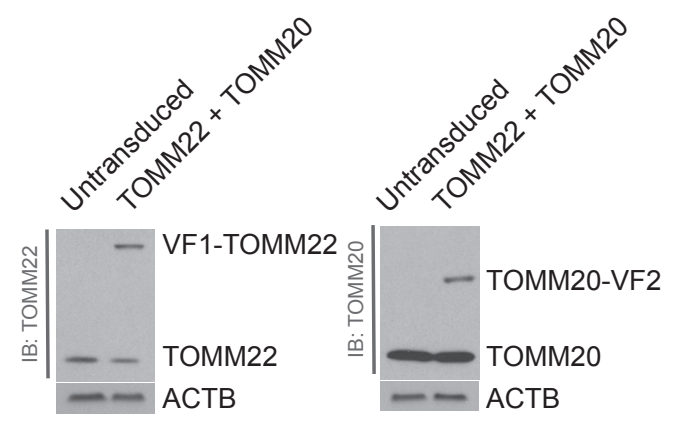

C

VF1-TOMM22
Expression Clone

MaGiCaL-PCA Library

Expression Clones

(1)

VF1 TOMM22

VF2 Preys

(2)

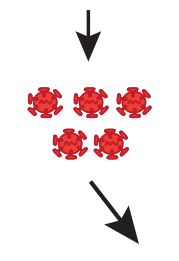

Replica 1

Replica 2

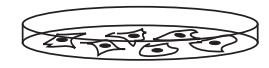

Replica 3

OR Preys VF2

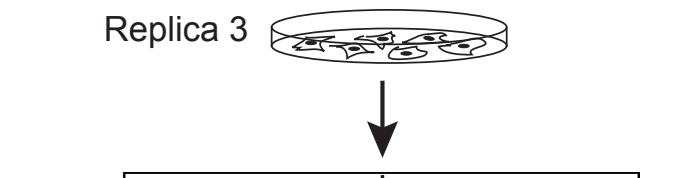

(3)

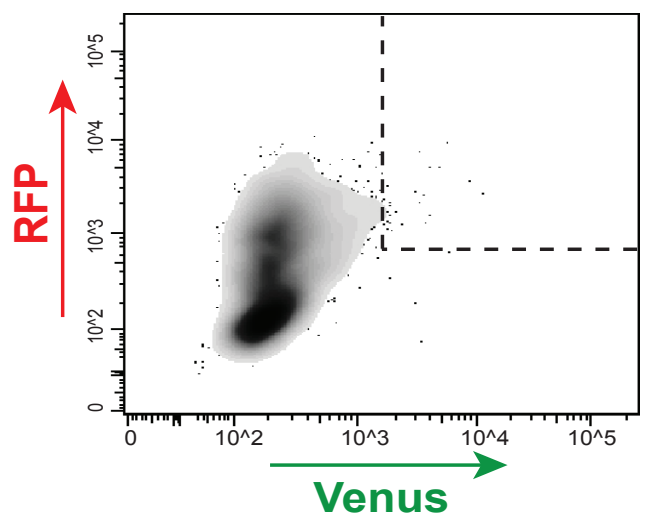

(4) Genomic DNA extraction, cDNA amplification, labeling and hybridization
B

D

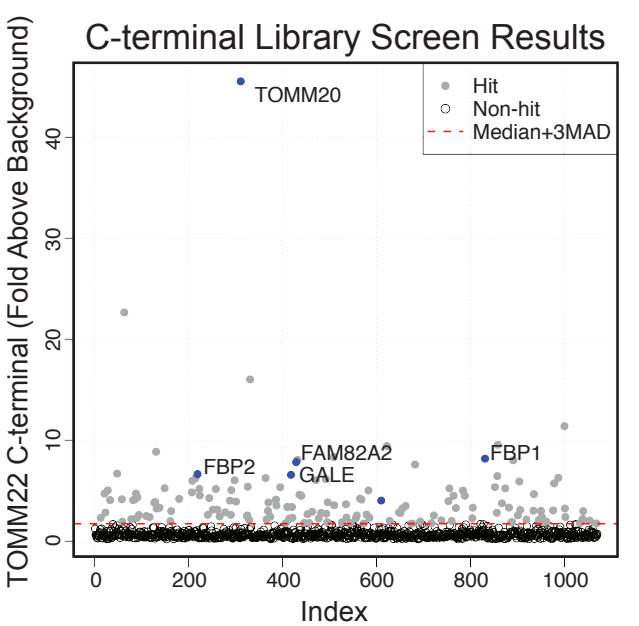

E

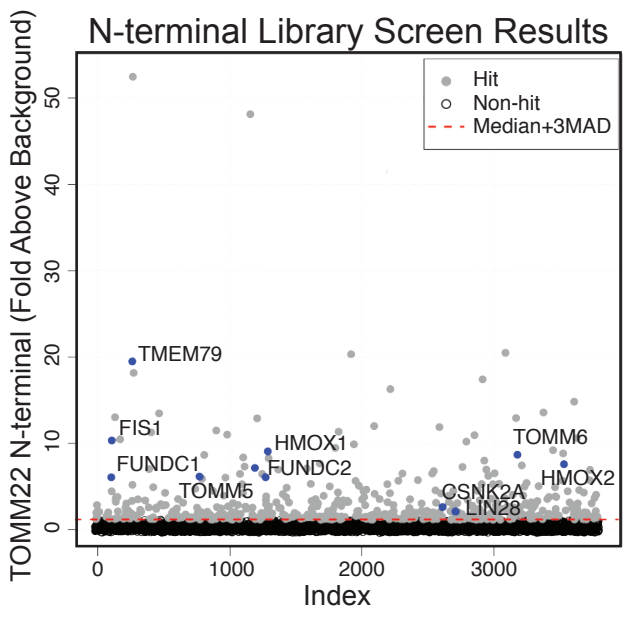


bioRxiv preprint doi: https://doi.org/10.1101/770669; this version posted September 16, 2019. The copyright holder for this preprint (which was

not certified by peer review) is the author/funder, who has granted bioRxiv a license to display the preprint in perpetuity. It is made available under aCC-BY-NC-ND 4.0 International license.

Figure 3

A

PCA signal (VF1-TOMM22 + prey)
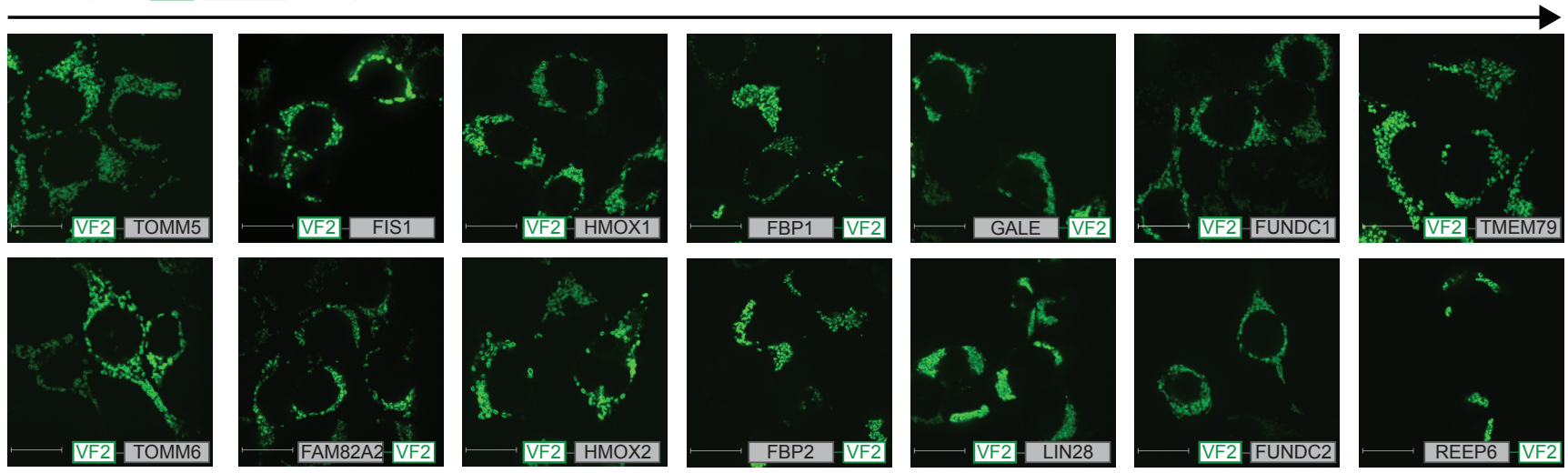

\section{Predicted \\ Interactors}
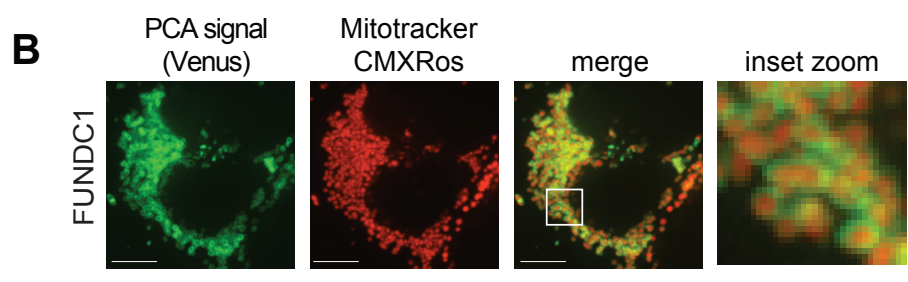

Interactions identified in

PCA screen
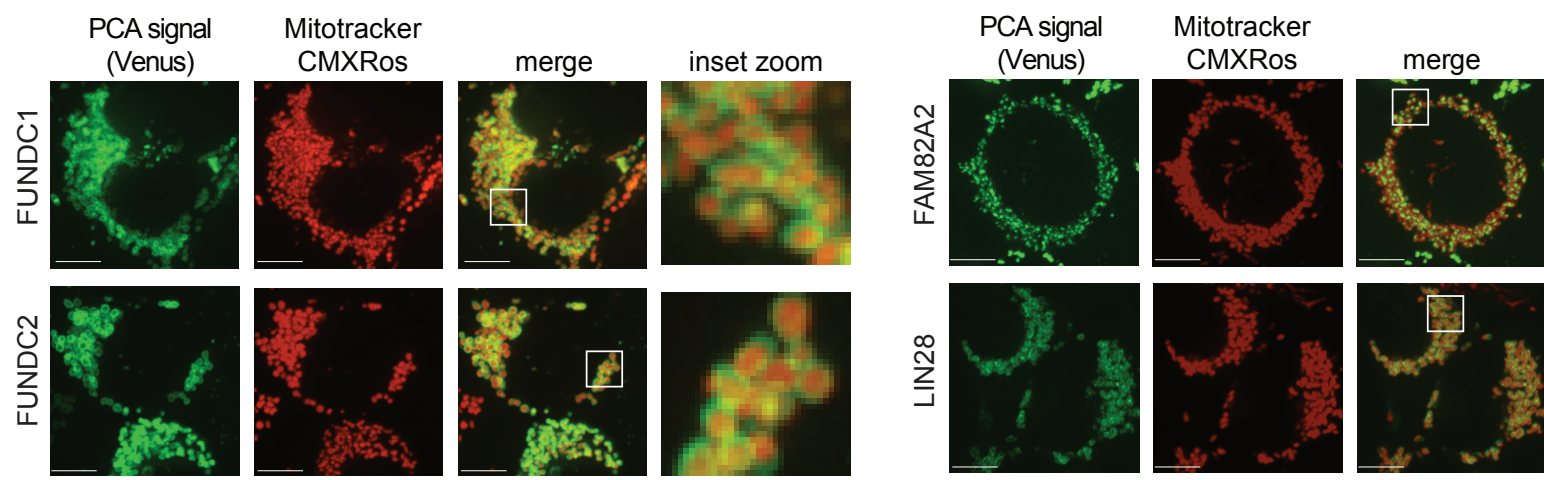

inset zoom
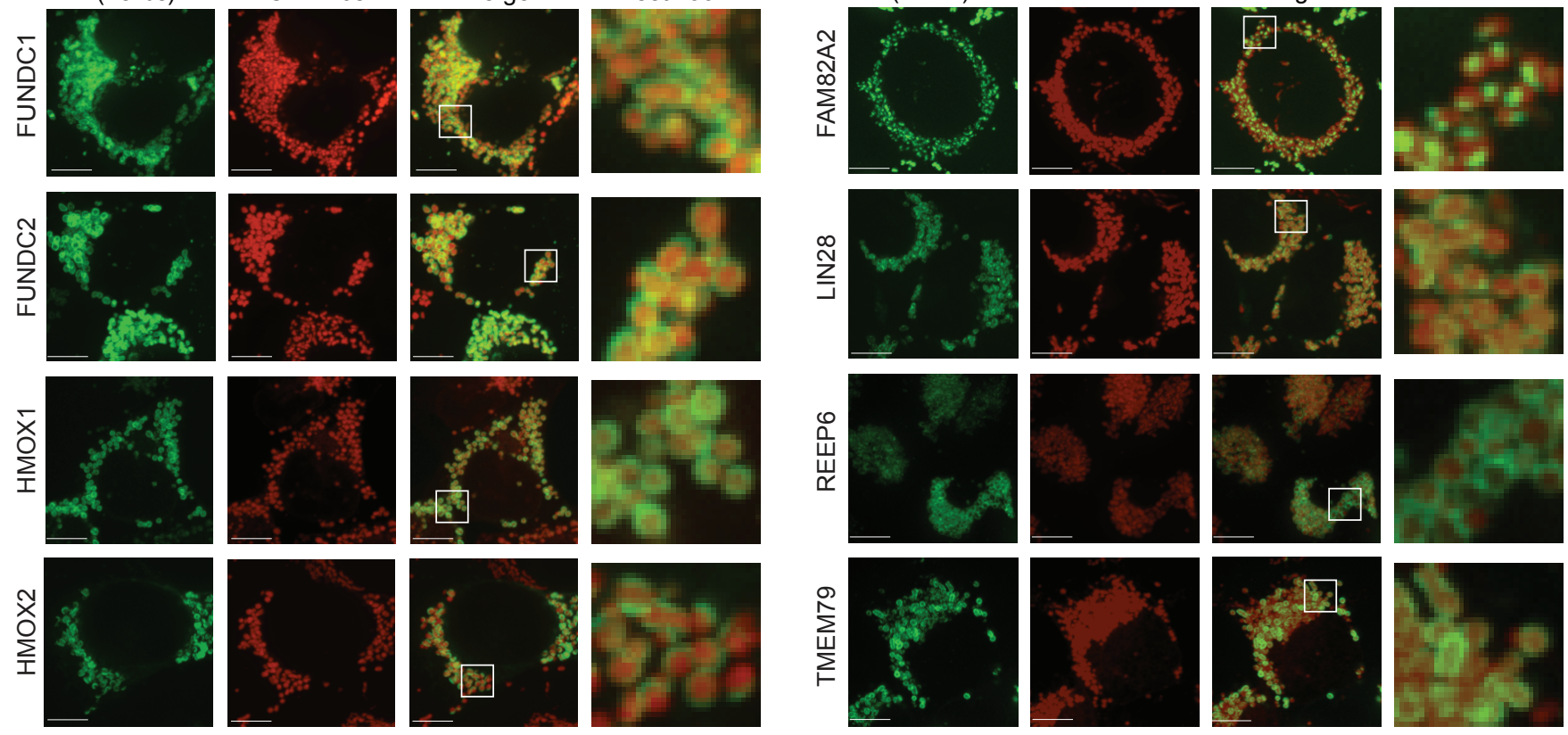
B

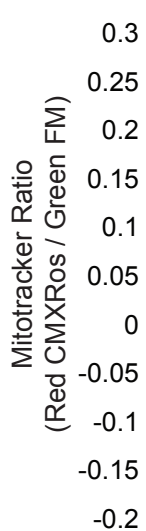

C

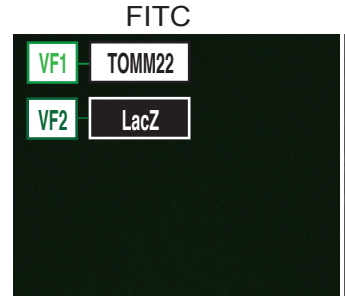

\section{VF1 TOMM22 \\ VF2 CSNK2A1}

E
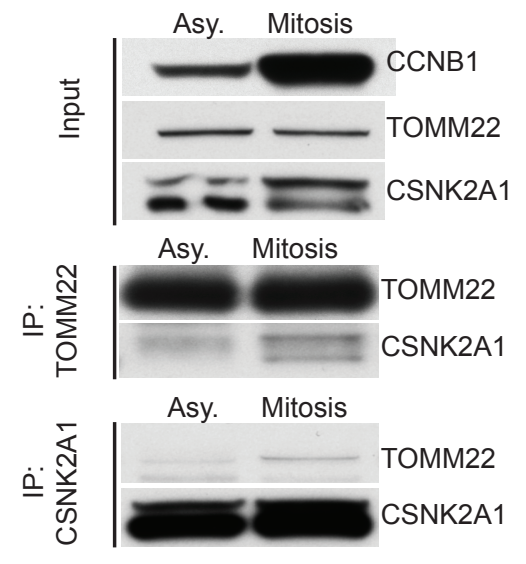

FITC / BF

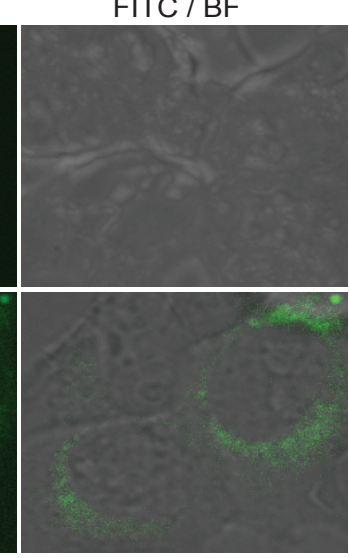

D

MYC-GFP

MYC-CSNK2A1

MYC-TOMM34

FLAG-TOMM22

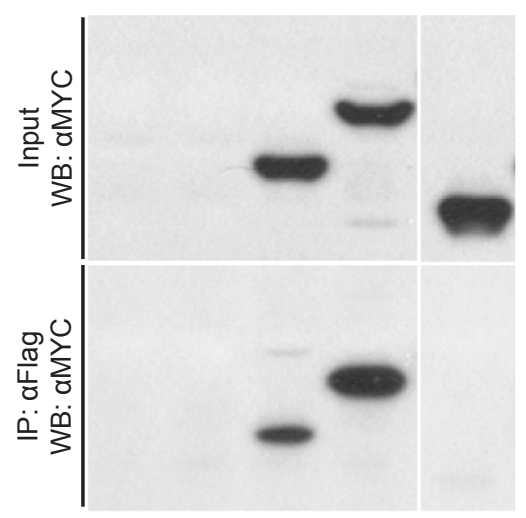

Image with InCell Analyzer 2000
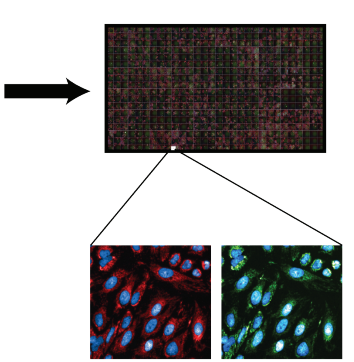

CSNK2A 1 MFN2

Genes 
bioRxiv preprint doi: https://doi org/10.1101/770669; this version posted September 16, 2019. The copyright holder for this preprint (which was not certified by peer review) is the author/funder, who has granted bioRxiv a license to display the preprint in perpetuity. It is made available under aCC-BY-NC-ND 4.0 International license.

Figure 5

A

\begin{tabular}{|c|c|c|c|c|c|c|c|}
\hline Time (min) & 30 & 0 & 5 & 15 & 30 & 30 & \\
\hline CSNK & - & + & + & + & + & + & \\
\hline \multirow[t]{4}{*}{ TOMM22 } & + & + & + & + & + & - & \\
\hline & & & 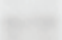 & $z^{2}=$ & -0 & 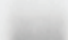 & $\leftarrow$ TOMM22 \\
\hline & & & 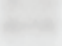 & $-1=$ & -10 & $=$ & $-\mathrm{CSNK} 2 \mathrm{~A} 1$ \\
\hline & & & 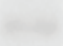 & $=$ & $-\infty$ & $=$ & $-\mathrm{CSNK} 2 \mathrm{~B} 1$ \\
\hline
\end{tabular}

B

pS15 pT43

\begin{tabular}{l|l} 
Cytoplasmic & TM \\
\hline
\end{tabular}

IMS

ShTOMM22

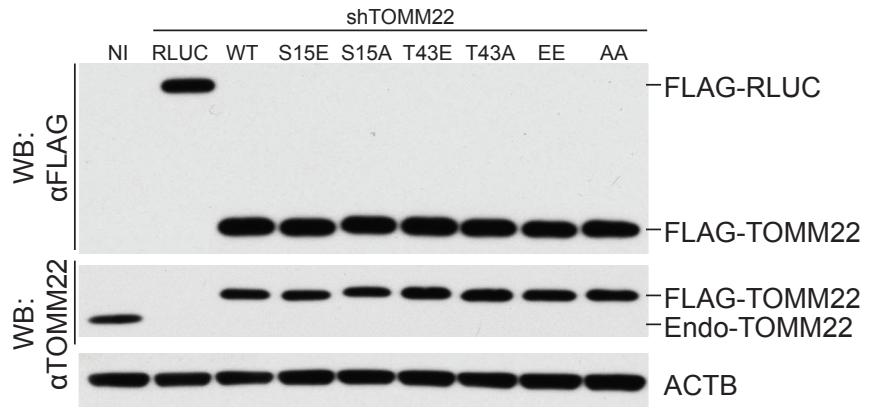

C

RLUC WT S15E S15A T43E T43A EE AA

FLAG-TOMM22

$\longrightarrow-\infty=0-\mathrm{ACTB}$

D

RLUC WT S15E S15A T43E T43A EE AA

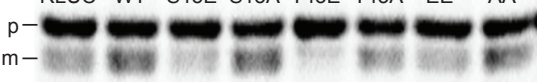

$\begin{array}{lllllllll}34.7 & 50.5 & 28.8 & 54.6 & 34.4 & 42.2 & 31.9 & 45.0 & \%\end{array}$

E

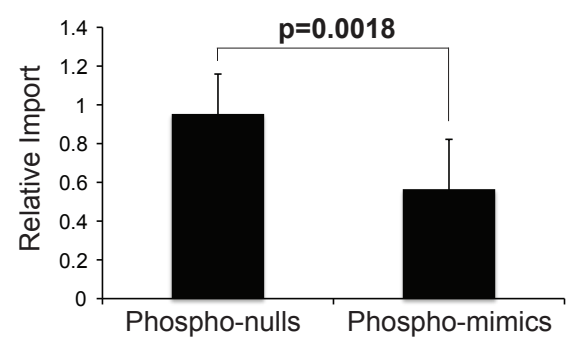

